\title{
The Effect of Different Plaque Control Modalities on the Keratinizing Potential of the Sulcular Epithelium in Monkeys*
}

\author{
Fred L. Bye, $†$ Raul G. Caffesse, $\ddagger$ and Carlos E. Nasjleti§
}

\begin{abstract}
THE PURPOSE OF this study was to determine if various plaque control regimes would be effective in permitting the gingival sulcular epithelium to keratinize. Three adult Rhesus monkeys had their teeth scaled and were placed on specific plaque control regimes including various combinations of prophylaxes, topical chlorhexidine and systemic tetracycline. Over the 2-month experimental period, weekly Plaque and Gingival Indices were determined. Subgingival plaque samples were obtained during the first 2 treatment weeks and at the end of the 1st month. Block sections of individual teeth from the different experimental regimes were serially sectioned to be evaluated histologically for inflammation and keratinization. Plaque and Gingival Indices were significantly reduced by the end of the 8th week compared to the pretreatment levels for all experimental regimes, except that the regime consisting only of three prophylaxes per week failed to reduce the Gingival Index significantly. Rubber cup prophylaxes were less effective in reducing plaque and inflammation than when the prophylaxes were combined with topical chlorhexidine application or systemic tetracycline administration. However, all experimental regimes were capable of reducing the subgingival bacterial flora to a level permitting sufficient reduction in gingival inflammation so that sulcular keratinization would occur. Some degree of sulcular keratinization was observed for every tooth of all experimental regimes. All regimes caused a significant decrease in the Inflammatory Indices relative to the control values. A negative correlation was found between the Crestal Inflammatory Index and the Keratin Width and Keratin Length, while a positive correlation was apparent between the Keratin Width and Keratin Length.
\end{abstract}

Keratinized stratified squamous epithelium undergoes continual replacement by an orderly process involving cell migration and differentiation through four discrete morphologic stages of development. The final surface layer of keratin is generally thought to be a protective barrier.

The lack of a keratinized layer for the oral sulcular epithelium may be important relative to the pathogenesis of periodontal disease, since the absence of an effective barrier may relate to the suggested process of bacterial products entering the gingival tissues and initiating gingival inflammation. Recently, the keratinizing potential of the sulcular epithelium has been demonstrated by the surgical displacement of the sulcular tissue from approx-

\footnotetext{
* This study was partially supported by the Medical Research Division of the Veterans Administration.

$\dagger$ Present address: U.S.A.F. Regional Hospital, Shaw Air Force Base, South Carolina 29152. Formerly Department of Periodontics, The University of Michigan School of Dentistry, Ann Arbor, MI 48109.

$\ddagger$ Professor and Chairman, Department of Periodontics, The University of Michigan School of Dentistry, Ann Arbor, MI 48109.

$\S$ Dental Research Section, Veterans Administration Hospital, Ann Arbor, MI 48105.
}

imating the tooth. ${ }^{1,2}$ Furthermore, intensive antibacterial therapy has been shown to permit in situ keratinization of the sulcular epithelium. ${ }^{3}$

This study investigated the effect of different treatment modalities for plaque control on the keratinizing potential of the sulcular epithelium.

\section{MATERIALS AND METHODS}

Three adult Rhesus monkeys (macaca mulatta) were used for the investigation. Two were females, with Monkey 1 weighing $11.0 \mathrm{lb}(4.990 \mathrm{~kg})$ and Monkey 2 weighing $10.5 \mathrm{lb}(4.763 \mathrm{~kg})$. Monkey 3 was a male weighing 19.5 lb $(8.168 \mathrm{~kg})$. All had nearly complete dentitions. Oral radiographic analysis demonstrated similar levels and health status of the supporting bone.

The monkeys were sedated with ketamine hydrochloride intramuscularly (IM) $(25 \mathrm{mg} / \mathrm{kg})$ and anesthetized with sodium pentobarbital intravenously (IV), $(50 \mathrm{mg}$ / $\mathrm{ml}$ ). A pretreatment oral clinical examination included an assessment of the gingival condition according to the Gingival Index System. ${ }^{4}$ Soft deposits were assessed according to the Plaque Index System. ${ }^{5}$ Also, pretreatment subgingival plaque samples were obtained from 
specific sample sites of representative quadrants using a sterile No. 13-14 Columbia* curette. Subsequent plaque samples were obtained from the same locations.

After the first clinical examination all teeth were thoroughly scaled and polished. On the day of the initial prophylaxis all animals were given IV injectable tetracycline hydrochloride (Achromycin ${ }^{\circledR}$ ) at the maximum safe dosage of $20 \mathrm{mg} / \mathrm{kg}$ body weight. Monkeys 1 and 2 were given the same dosage daily for 1 week only, while Monkey 3 was maintained on its same initial dosage daily throughout the 2-month investigation period.

All treatment regimes were conducted during the 5 weekdays only, except for the tetracycline administration which was not interrupted. Monkeys 1 and 2 began their appropriate treatment regimes on day 4 of the initial week-long tetracycline program and continued on that same regime for 2 months. During this investigation period the following experimental regimes were adhered to:

Monkey 1 (1 week only, IV tetracycline hydrochloride)

CP5 maxillary and mandibular right quadrants: daily prophylaxis and daily topical application of $0.2 \%$ chlorhexidine gluconate

CP1 maxillary and mandibular left quadrants: 1 prophylaxis weekly and daily topical application of $0.2 \%$ chlorhexidine gluconate

Monkey 2 (1 week only, IV tetracycline hydrochloride)

P5 maxillary and mandibular right quadrants: daily prophylaxis only

P3 maxillary and mandibular left quadrants: prophylaxis 3 times per week

Monkey 3 began its treatment regime on day 4 of the continuous tetracycline program and remained on the same regime for 2 months. During this investigation period the following experimental regime was adhered to:

Monkey 3 (continuous daily IV tetracycline hydrochloride)

TP5 maxillary and mandibular right quadrants: daily prophylaxis only

TP1 maxillary and mandibular left quadrants: 1 prophylaxis weekly

During the experimental period, for any treatment or index scoring procedures, all animals were premedicated with ketamine hydrochloride ( $25 \mathrm{mg} / \mathrm{kg}$ IM) to insure cooperation. The scheduled prophylaxes consisted of a rubber cup polish with prophylaxis paste (Nupro®). The $0.2 \%$ chlorhexidine gel application was accomplished using a $10 \mathrm{cc}$ syringe with a 22 gauge irrigation needle to apply a continuous bead of chlorhexidine both facially and lingually to the tooth surface along the free gingival margin. The areas to receive the topical chlorhexidine were first isolated with gauze squares and kept free of saliva. A total of approximately $2 \mathrm{cc}$ was used for each

* Star Dental Manufacturing Company, Conshohocken, PA. complete mouth application. The chlorhexidine was not washed off after application.

On the last day of each week any treatment procedures were preceded by a scoring of all the monkeys, first for the Plaque Index and then for the Gingival Index. Index measurements were made for all teeth, both facially and lingually, at the mesial, midpoint and distal aspects. To determine the pretreatment and weekly Plaque Indices of each experimental regime, the six scores for each tooth were first averaged separately, and the means of all teeth for a particular regime were then averaged to get the overall Plaque Index value from that regime. The six Gingival Index scores for each tooth were treated in an identical manner to arrive at the overall Gingival Index for a particular experimental regime.

At the end of week 1 (the completion of the tetracycline programs for Monkeys 1 and 2), and after 1 month, subgingival plaque samples were obtained from all monkeys, and processed by previously described procedures. ${ }^{6}$

After 2 months, the animals were sacrificed by exsanguination. Following decapitation, the heads were fixed in $10 \%$ buffered formalin. The jaws were later dissected and placed in $10 \%$ formic acid. The block sections were embedded in paraffin, were cut buccolingually into $6-\mu$ sections with a microtome, and mounted on glass slides. The slides were stained with either Ehrlich's acid hematoxylin and eosin, Mallory's trichrome stain or Rhodamine $B$.

\section{Histologic Evaluation}

For histologic evaluation, the gingival sulcular epithelium was divided in half, into two zones. The Crestal zone included the coronal one-half of the sulcular epithelium and its subjacent connective tissue (Fig. 1A). The Cervical zone was comprised of the remaining onehalf of the sulcular epithelium extending to the beginning of the junctional epithelium.

A histologic determination was made of the distribution of keratin within the sulcular area. Furthermore, a quantitative analysis of the Keratin Width and Keratin Length was performed using the Mallory's trichrome stained sections and a Filar Micrometer Eyepiece (Bausch and Lomb®). Keratin Widths (Fig. 1B) were measured for all available sections prepared with Mallory's trichrome stain. Width measurements were made separately for the buccal and lingual sulcular areas at the approximate midpoint of the sulcular keratin band. For each tooth within a particular experimental regime, measurements for the individual sections were summed and averaged both buccally and lingually. An overall buccal or lingual Keratin Width value for a given regime was then determined by averaging the calculated means of the individual teeth within that regime. A combined buccal and lingual Keratin Width value could be found also for each regime by computing the average of the buccal and lingual Keratin Width values. Keratin 

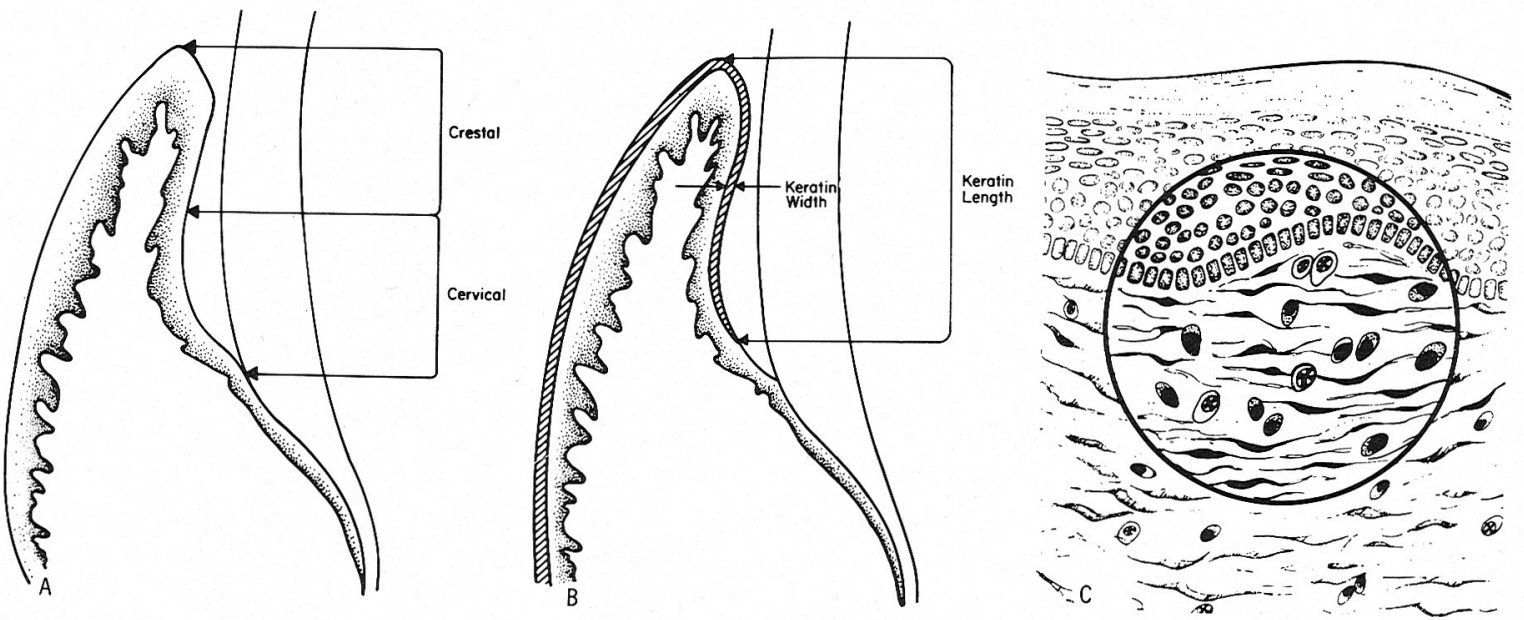

Figure 1A. Diagram showing the zones of the gingival sulcus in which the Crestal Inflammatory Index and Cervical Inflammatory Index were determined. B. Diagram showing the manner in which Keratin Width and Keratin Length were measured. C. Diagrammatic representation of the microscopic area selected for determination of the Inflammatory Indices.

Lengths (Fig. 1B) were also measured for all available sections prepared with Mallory's trichrome stain. Length measurements were made separately for the buccal and lingual sulcular areas and mean Keratin Length values for the various regimes were determined like those for Keratin Widths.

All slides stained with Ehrlich's acid hematoxylin and eosin were evaluated for severity of inflammation in the connective tissue subjacent to the Crestal and Cervical sulcular epithelial zones. An Inflammatory Index was determined for each zone for purposes of quantitation by counting the number of inflammatory cells within a microscopic field. The microscopic field $(400 \times)$ was positioned so that the basal cell layer divided the field into two segments, with the connective tissue occupying approximately two-thirds of the field and the epithelium occupying the remaining one-third (Fig. 1C). Separate Crestal and Cervical Inflammatory Indices were determined both buccally and lingually for the different experimental regimes in a manner identical to that discussed above for calculating Keratin Widths and Keratin Lengths. Control Crestal and Cervical Inflammatory Indices were also determined using hematoxylin and eosin stained sections (Fig. 2) obtained from another monkey which had never received antibacterial therapy of any kind.

\section{Statistical Analysis}

Data were analyzed by the Michigan Interactive Data Analysis System (MIDAS) of the University of Michigan Statistical Research Laboratory using analysis of variance, profile analysis, paired $t$ tests, two-sample $t$ tests and product-moment correlation coefficients.

\section{RESULTS}

\section{Clinical Findings}

Plaque Index. No statistically significant difference was found between the pretreatment Plaque Index scores

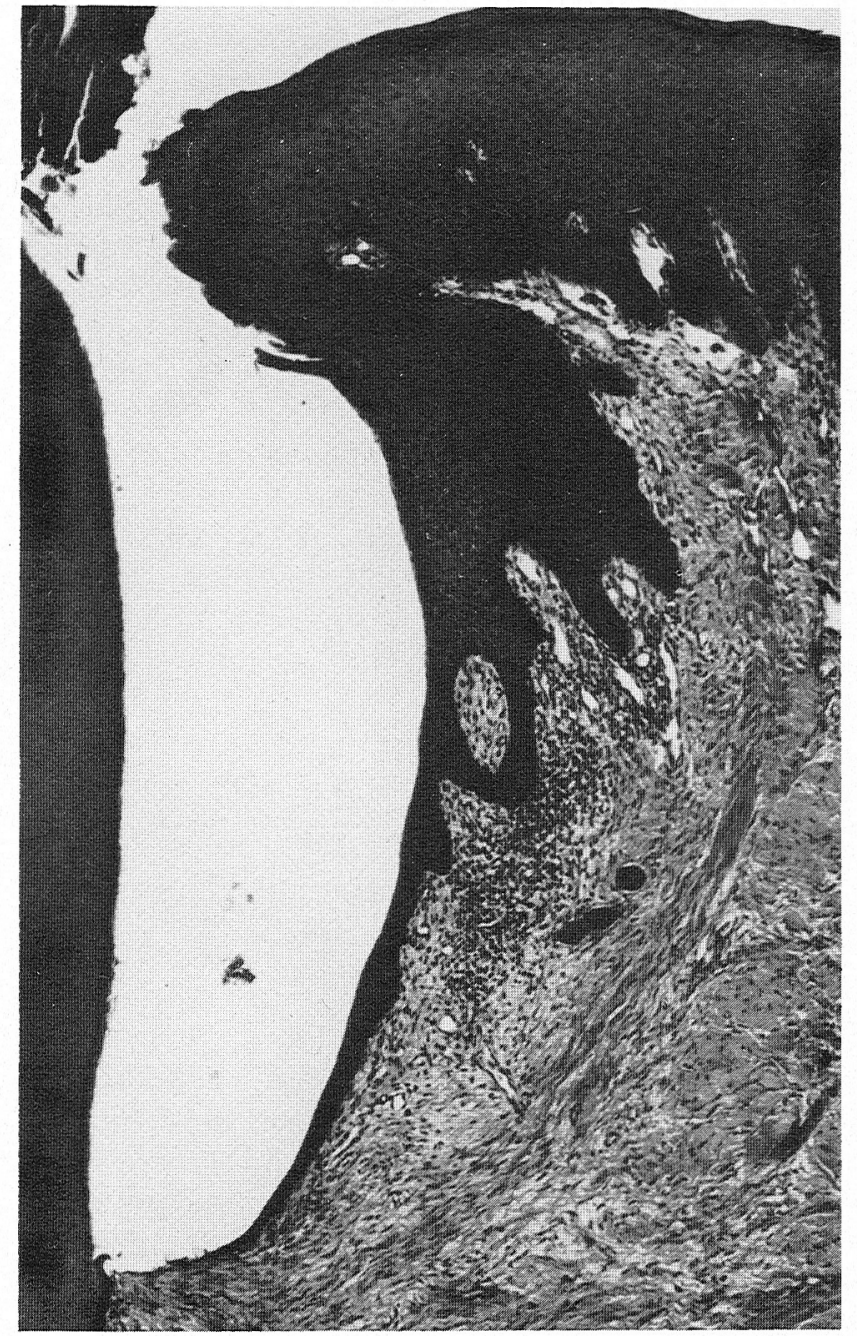

Figure 2. Gingival sulcus of monkey prior to any antibacterial treatment $(H \&$ E, original magnification, $\times 100)$.

for the different experimental regimes. There was a statistically significant difference $(P<0.01)$ between the pretreatment Plaque Index values and the values determined at the completion of the 8-week investigation 


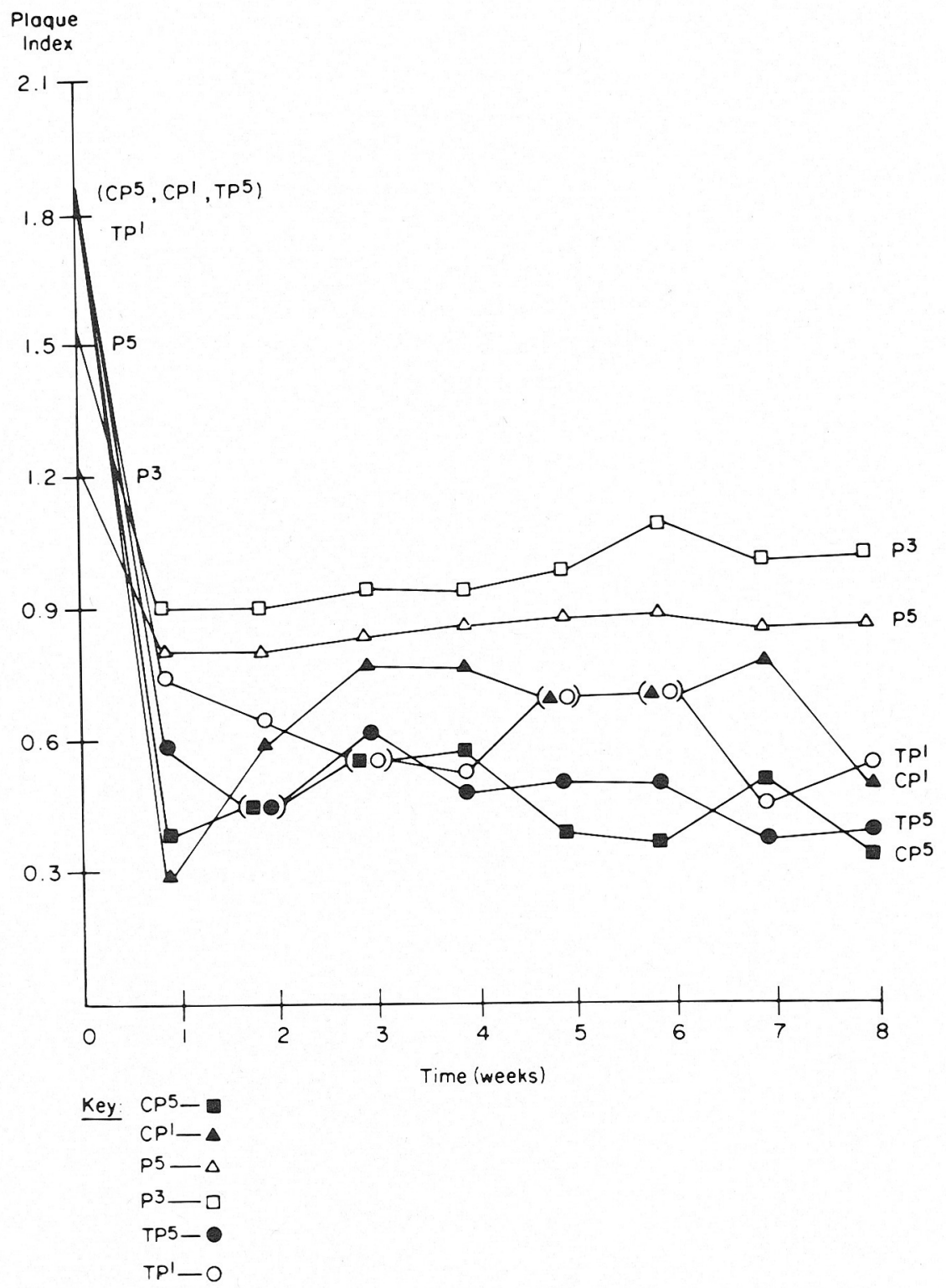

Figure 3. Profile analysis plot of mean Plaque Indices from different experimental regimes.

period for all experimental regimes. Over the 8 weeks, the Plaque Index time trend for each experimental regime was significantly different from the trend for each of the others (Fig. 3). The week 8 Plaque Index for regime $\mathrm{P} 3$ was the highest of all the experimental regimes and was significantly different $(P<0.05)$ from each of the other experimental regimes, except regime P5 (Fig. 5A). Regime P5 had the second highest Plaque Index at week 8 , which differed significantly $(P<0.05)$ from the two experimental regimes having the lowest Plaque Index values. No other significant differences were found between the experimental regimes for the Plaque Index values at week 8 .

Gingival Index. No statistically significant difference was found between the pretreatment Gingival Index scores for the different experimental regimes. There was a significant difference $(P<0.01)$ between the pretreatment Gingival Index values and the values determined at the completion of the 8-week investigation period for all experimental regimes except regime $\mathrm{P} 3$ which showed a borderline significance. The Gingival Index time trends closely approached one another by the second treatment week (Fig. 4). After the 2nd week, however, the Gingival Index time trends for the different experimental regimes were not parallel to each other. The week 8 Gingival Index for regime P3 was the highest of all experimental regimes and was significantly different $(P<0.05)$ from each of the other experimental regimes (Fig. 5B). At week 8 regime $\mathrm{P} 5$ had the second highest Gingival Index, which differed significantly $(P<0.05)$ from the two experimental regimes having the lowest Gingival Index values. No other significant differences existed between the experimental regimes for the Gingival Index values at week 8 .

Bacteriology. For each experimental regime there was a dramatic reduction in the total count of microorga- 


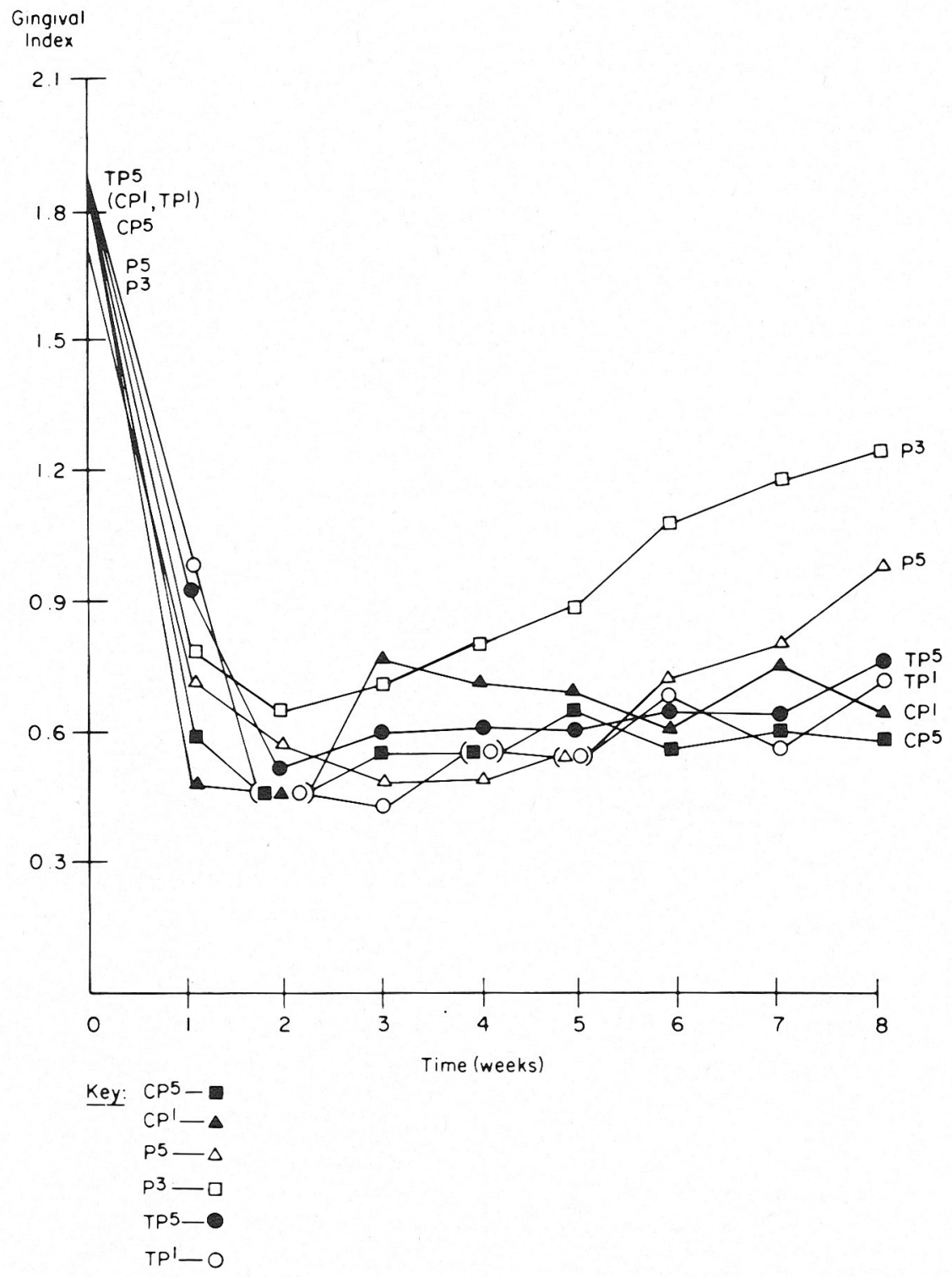

Figure 4. Profile analysis plot of mean Gingival Indices from different experimental regimes.

nisms after 4 weeks. While the week 4 values for regimes CP5, CP1, TP5 and TP1 were consistent with or lower than the week 1 values, regimes $\mathrm{P} 5$ and $\mathrm{P} 3$ showed much higher values after 4 weeks than at week 1 . The ratio of anaerobes to aerobes was also markedly decreased for each of the experimental regimes at the end of the 4th week, with much of the reduction having occurred by the 1st week. The percentage of Actinomyces viscosus remained at zero after 4 weeks for regimes CP5, CP1 and TP5 but increased by the 4th week for the remaining experimental regimes. The percentage of Actinomyces naeslundii increased over the 4 weeks for all experimental regimes except regime $\mathrm{CP} 5$, where a slight reduction occurred. After 4 weeks, all regimes had a low or zero percentage of Bacteroides melaninogenicus. Fusobacteria increased in percentage for regimes P5 and P3 after 4 weeks, but were reduced for the remaining experimental regimes. The percentage of Bacteroides Ochraceus after 4 weeks was zero for regimes CP5, CP1, TP5 and TP1 but had increased for regimes P5 and P3. Compared to the pretreatment percentages the percentages of Streptococcus sanguis increased markedly by the 4 th week for all regimes, with the exception of regimes P5 and P3.

\section{Histologic Findings}

Histologic Description. Some degree of sulcular keratinization was observed for every tooth of all experimental regimes. Orthokeratinization of the gingival sulcular epithelium was most generally observed, although a few cases of parakeratinization were found. The extent to which the sulcular epithelium keratinized in an occlusalcervical direction (i.e. the Keratin Length) varied among the experimental regimes, ranging from keratinization restricted to the occlusal portions of the gingival sulcus to an intact band of keratin extending the entire length of the sulcus (Figs. 6A, 6B, and 6C).

Inflammatory Index. For all experimental regimes, the mean Crestal Inflammatory Indices (Table 1, Fig. 6B) 
and mean Cervical Inflammatory Indices (Table 2, Fig. 6B) were significantly different $(P<0.001)$ from the corresponding Control Inflammatory Indices (Tables 1 and 2, Fig. 2). In the buccal Crestal Inflammatory Indices there were significant differences $(P<0.05)$ only between the experimental regime having the lowest index value and the two experimental regimes of highest index values (Fig. 7a). In the lingual Crestal Inflammatory Indices there were significant differences $(P<0.05)$ between the

a) Plaque Indices

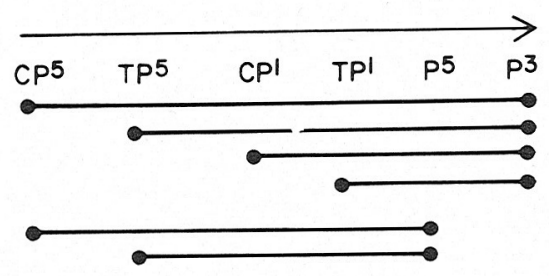

b) Gingival Indices*
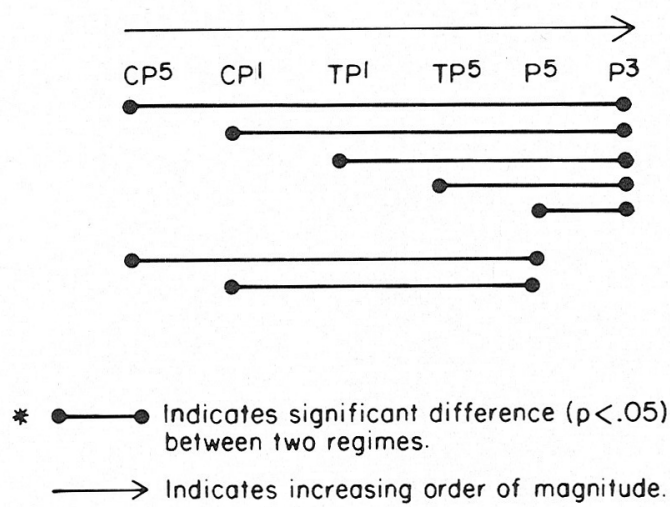

Figure 5. Comparison of experimental regimes by analysis of variance of week 8 mean. experimental regime with the highest index value and the four experimental regimes with the lowest values, as well as between the regime with the second highest Inflammatory Index and the two regimes with the lowest index values (Fig. 8a). In the combined buccal and lingual Crestal Inflammatory Indices there were significant differences $(P<0.05)$ between the experimental regime with the highest index value and the four experimental regimes having the lowest index values, as well as between the regime with the second highest Inflammatory Index and the three regimes with the lowest values (Fig. 9a).

Keratin Width. Buccal Keratin Widths were significantly different $(P<0.05)$ only between the experimental regime with the lowest width value and the two experimental regimes having the highest width values (Fig. $7 b)$. Significant differences $(P<0.05)$ in Keratin Widths of the lingual sulcular areas were apparent between the experimental regime having the greatest width and the three regimes with lowest width measures (Fig. 8b). Also, there were significant differences in lingual Keratin Width between the second highest value regime and the two lowest value regimes. Buccal and lingual Keratin Widths were significantly different $(P<0.01)$ from each other for experimental regimes P5, P3 and TP5 only (Table 3). Significant differences for combined buccal and lingual Keratin Widths were apparent between the experimental regimes having the greatest width and the three regimes with the lowest width measures (Fig. 9b). There were also significant differences in combined Keratin Widths between the second highest value regime and the three lowest value regimes.

Keratin Length. There was no significant difference among any of the experimental regimes in buccal Keratin Length (Fig. 7c) or in lingual Keratin Length (Fig. 8c). No significant difference was found in the buccal and lingual keratin lengths among any of the experimental
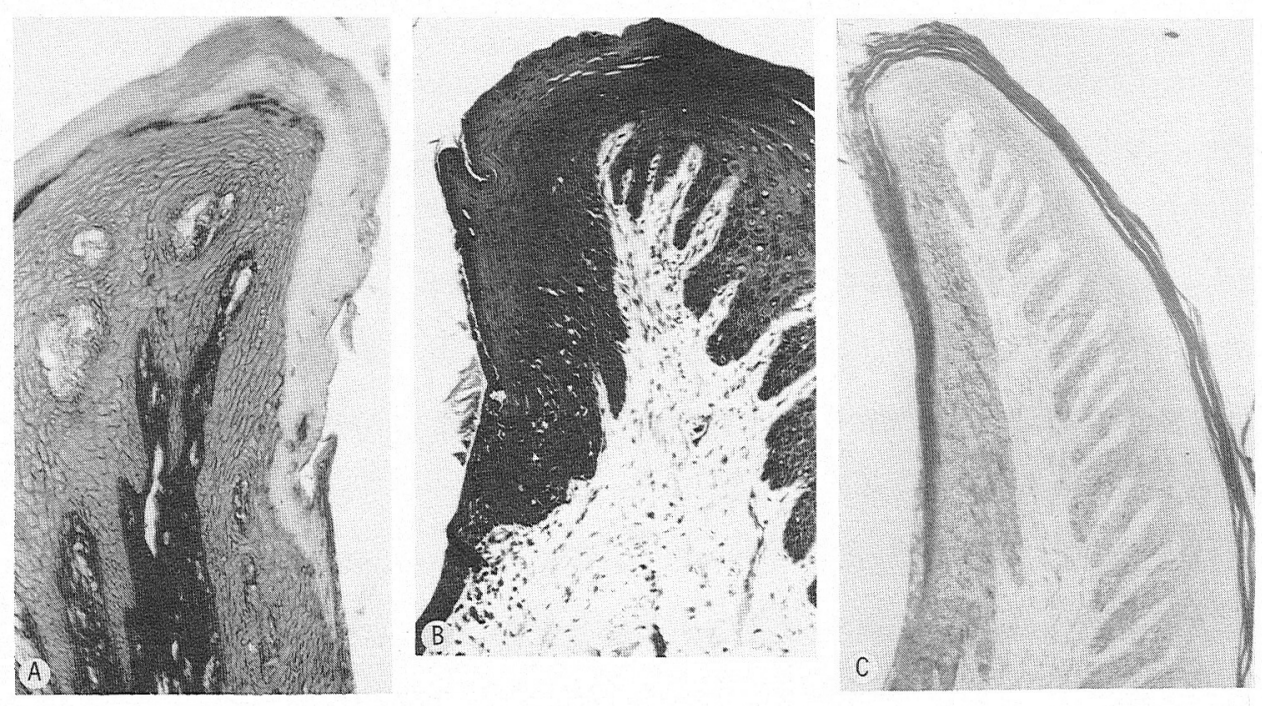

Figure 6A. Thick keratin band covering sulcular epithelium (Mallory Trichrome stain, original magnification, $\times 100)$. B. Parakeratinization of sulcular epithelium $(H \& E$, original magnification, $\times 100)$. C. Thick keratin band extending entire length of sulcus (Rhodamine B stain, original magnification, $\times 100)$. 
Table 1

Two-Sample $t$ Tests Comparing Mean Control and Experimental Crestal Inflammatory Indices

\begin{tabular}{ccccccc}
\hline Control mean & N & $\begin{array}{c}\text { Experimental } \\
\text { regime }\end{array}$ & N & Mean & Variance & Significance \\
\hline 74.9 & 14 & CP5 & 13 & 21.5 & 43.8 & 0.0000 \\
& CP1 & 14 & 22.3 & 36.9 & 0.0000 \\
& P5 & 14 & 6.8 & 3.2 & 0.0000 \\
& P3 & 13 & 14.0 & 53.2 & 0.0000 \\
& TP5 & 12 & 10.4 & 40.1 & 0.0000 \\
& TP1 & 12 & 10.6 & 22.1 & 0.0000 \\
\hline
\end{tabular}

Table 2

Two-Sample $t$ Tests Comparing Mean Control and Experimental Cervical Inflammatory Indices

\begin{tabular}{ccccccc}
\hline Control mean & $\mathrm{N}$ & $\begin{array}{c}\text { Experimental } \\
\text { regime }\end{array}$ & $\mathrm{N}$ & Mean & Variance & Significance \\
\hline 132.3 & 14 & CP5 & 13 & 35.4 & 637.7 & 0.0000 \\
& & CP1 & 14 & 30.5 & 441.5 & 0.0000 \\
& P5 & 14 & 39.6 & 1263.7 & 0.0000 \\
& P3 & 13 & 55.2 & 1672.1 & 0.0005 \\
& TP5 & 12 & 59.0 & 1471.0 & 0.0009 \\
& TP1 & 12 & 33.0 & 641.0 & 0.0000 \\
\hline
\end{tabular}

a) Buccal Crestal Inflammatory Indices*

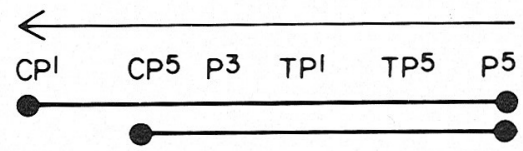

b) Buccal Keratin Widths*

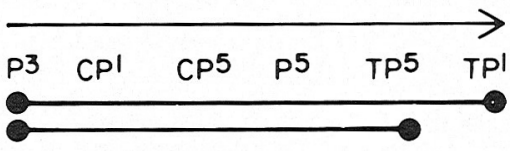

c) Buccal Keratin Lengths*

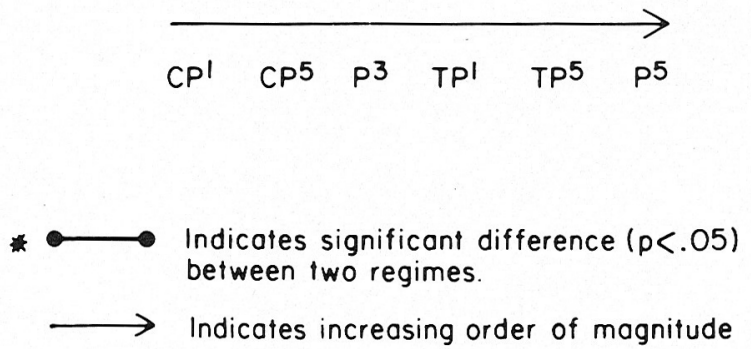

Figure 7. Comparison of experimental regimes by analysis of variance of mean.

regimes (Table 4). For the combined buccal and lingual Keratin Lengths there was a significant difference $(P<$ 0.05 ) only between the experimental regimes of highest and lowest values (Fig. 9c).
Correlations. The three experimental regimes with the lowest buccal Crestal Inflammatory Indices corresponded to the regimes demonstrating the three highest buccal Keratin Width and Keratin Length values (Figs. $7 \mathrm{a}, 7 \mathrm{~b}, 7 \mathrm{c}$ ). With the buccal Crestal Inflammatory Indices arranged in increasing order of magnitude for the experimental regimes, an inverse relationship was found compared to a similar ordering of buccal Keratin Lengths (note direction of arrows in Figs. 7a, 7c). The three experimental regimes with the lowest lingual Crestal Inflammation Indices corresponded to the regimes demonstrating the three highest lingual Keratin Width and Keratin Length values (Figs. 8a, 8b, 8c). With the combined buccal and lingual Crestal Inflammatory Indices of the experimental regimes arranged in increasing order of magnitude, an inverse relationship was found compared to a similar ordering of the combined Keratin Widths or Keratin Lengths (Figs. 9a, 9b, 9c). A negative correlation was found between the combined Crestal Inflammatory Indices and the combined Keratin Widths $(P<0.05)$ and combined Keratin Lengths (Table 5), while a positive correlation $(P<0.01)$ was apparent between the combined Keratin Widths and the combined Keratin Lengths.

\section{DISCUSSION}

The results of the present study agree with previous demonstrations $s^{1-3,6,7}$ that with an altered sulcular environment, the sulcular epithelium shows a potential for keratinization.

Of the various experimental regimes, those involving only three or five prophylaxes per week were less effective in reducing Plaque and Gingival Indices than those combining prophylaxes with antibacterial agents. However, five prophylaxes per week did significantly reduce Plaque and Gingival Index values compared to pretreatment values, and three prophylaxes per week signifi- 
a) Lingual Crestal Inflammatory Indices*

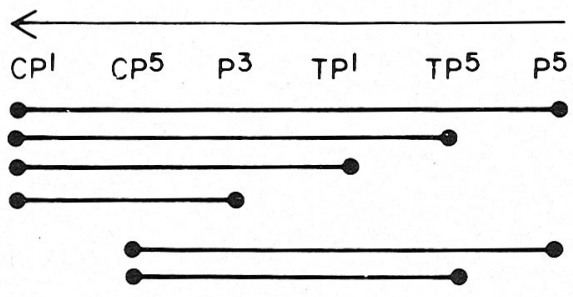

b) Lingual Keratin Widths*

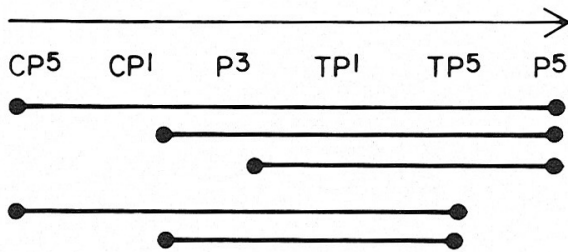

c) Lingual Keratin Lengths

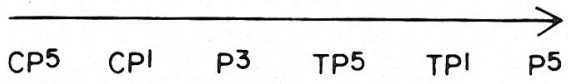

\footnotetext{
- $\longrightarrow$ Indicates significant difference $(p<.05)$ befween two regimes.
}

Indicates increasing order of magnifude

Figure 8. Comparison of experimental regimes by analysis of variance of mean.

cantly reduced Plaque Index values. The lack of a significant reduction in the Gingival Index by the 8 th week for the regime employing only three prophylaxes per week may be due partially to the fact that the pretreatment Gingival Index value for that regime was the lowest of all experimental regimes. However, all other experimental regimes had lower 8-week Gingival Index values than those for the three prophylaxes per week regime, which indicates that the improvement observed was more dramatic for those other regimes. The lack of a significant difference in the 8th week Plaque and Gingival Indices, for the regimes combining three or five prophylaxes per week with daily topical chlorhexidine or parenteral tetracycline, suggests that all antibacterial regimes tested are equally effective in reducing plaque deposition and decreasing gingival inflammation.

Bacteriologic sampling was done to compare the findings over the initial 4 weeks with those previously reported in a study of the effect of intensive antibacterial therapy on the bacteriology of the sulcular environment in monkeys. ${ }^{6}$ A close relationship was found between the results for the 1st week of treatment, during which time treatment regimes for the two studies were similar, with all animals receiving parenteral tetracycline. Thereafter, there was more variation in the bacterial flora changes, apparently due to the difference in therapeutic methods.

The role of connective tissue in genetically determining the specific tissue differentiation of the overlying epithelium has been adequately demonstrated. ${ }^{8,9} \mathrm{Be}$ sides, there is no apparent difference in composition of the connective tissue underlying the oral and the sulcular epithelium. In accordance with these findings, it has been demonstrated that the sulcular epithelium will keratinize when it is displaced from approximating the tooth. $^{1,2}$ Furthermore, it has been shown that the oral epithelium loses keratinization when positioned in contact with the tooth. ${ }^{7}$ It seems, therefore, that the tooth surface or the local sulcular environment may influence epithelial differentiation inhibiting keratinization.

It has been postulated that the passage of cells from the basal zone to the epithelial surface involves a timedependent maturation process. ${ }^{10,11}$ Any alteration in the

a) Combined Crestal Inflammatory Indices*

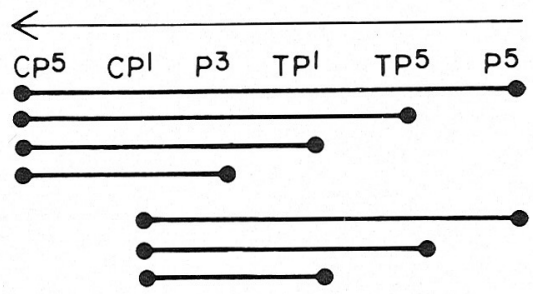

b) Combined Keratin Widths *

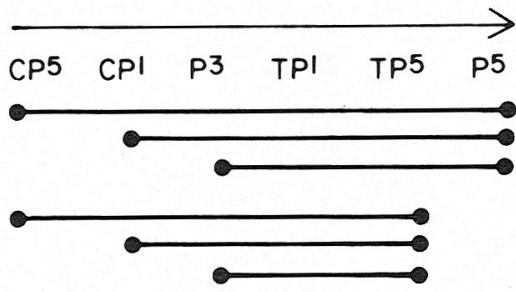

c) Combined Keratin Lengths *
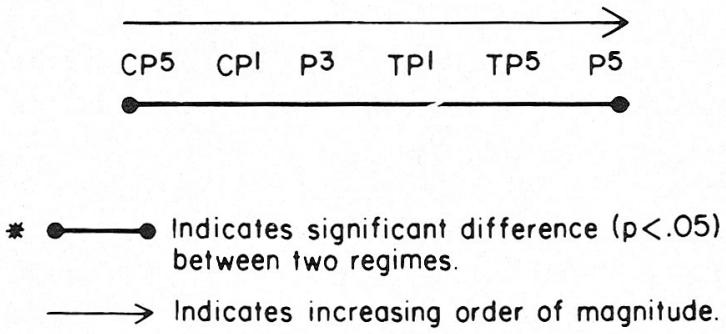

Figure 9. Comparison of experimental regimes by analysis of variance of mean. 
Table 3

Pairwise $t$ Test Comparing Mean Buccal and Lingual Keratin Widths for Different Experimental Regimes

\begin{tabular}{ccccccc}
\hline $\begin{array}{c}\text { Experimental } \\
\text { regime }\end{array}$ & $\begin{array}{c}\text { Buccal } \\
\text { mean* }\end{array}$ & $\begin{array}{c}\text { Lingual } \\
\text { mean* }\end{array}$ & $\begin{array}{c}\text { Mean differ- } \\
\text { ence }\end{array}$ & $\begin{array}{c}\text { Standard de- } \\
\text { viation }\end{array}$ & $t$ Statistic & Significance \\
\hline CP5 & 282.4 & 274.6 & 7.8 & 165.0 & 0.1697 & 0.8680 \\
CP1 & 281.9 & 332.2 & -50.3 & 130.9 & -1.4394 & 0.1737 \\
P5 & 380.0 & 687.0 & -307.0 & 269.2 & -4.2664 & 0.0009 \\
P3 & 235.0 & 430.7 & -195.7 & 140.7 & -5.0146 & 0.0003 \\
TP5 & 437.8 & 625.6 & -187.8 & 189.7 & -3.4297 & 0.0056 \\
TP1 & 418.6 & 511.4 & -92.8 & 205.6 & -1.5640 & 0.1461 \\
\hline
\end{tabular}

${ }^{*}$ Mean $\left(\times 10^{-5} \mathrm{~mm}\right)$.

Table 4

Pairwise $t$ Test Comparing Mean Buccal and Lingual Keratin Lengths for Different Experimental Regimes

\begin{tabular}{ccccccc}
\hline $\begin{array}{c}\text { Experimental } \\
\text { regime }\end{array}$ & Buccal mean* & Lingual mean & Mean difference & $\begin{array}{l}\text { Standard } \\
\text { deviation }\end{array}$ & $t$ Statistic & Significance \\
\hline CP5 & 477.7 & 491.9 & -14.2 & 218.3 & -0.2337 & 0.8191 \\
CP1 & 440.1 & 583.5 & -143.4 & 305.0 & -1.7589 & 0.1021 \\
P5 & 673.2 & 761.4 & -88.2 & 167.4 & -1.9714 & 0.0703 \\
P3 & 533.6 & 651.2 & -117.6 & 230.1 & -1.8418 & 0.0903 \\
TP5 & 636.3 & 645.7 & -9.4 & 238.8 & -0.1353 & 0.8948 \\
TP1 & 598.8 & 664.5 & -65.7 & 307.4 & -0.7409 & 0.4742 \\
\hline
\end{tabular}

${ }^{*} \operatorname{Mean}\left(\times 10^{-3} \mathrm{~mm}\right)$

Table 5

Product-Moment Correlation Coefficients of Crestal Inflammatory Index, Keratin Width, Keratin Length

\begin{tabular}{|c|c|c|}
\cline { 2 - 3 } \multicolumn{1}{c|}{} & \multicolumn{2}{|c|}{ Crestal inflammatory index } \\
\hline \multirow{2}{*}{ Keratin width } & -0.3570 & -0.1366 \\
\cline { 2 - 3 } & 0.4491 & -12 \\
\hline
\end{tabular}

cell turnover rate therefore could affect epithelial cell differentiation. Taking into account the fact that the presence of a mucosal bacterial flora is associated with an increased turnover rate of the mucosal epithelium in the ileum, ${ }^{12}$ a recent investigation ${ }^{3}$ has demonstrated that an extreme reduction of the subgingival bacterial flora is accompanied by significant decreases in gingival inflammation and mitotic activity of the sulcus. These changes have been associated with a full differentiation of the epithelium resulting in keratinization of the sulcular epithelium.

The present study was designed to determine the effect of different plaque control modalities on the keratinizing potential of the sulcular epithelium. Each of the six experimental regimes tested permitted demonstrable keratinization of the gingival sulcus. All experimental regimes, therefore, were capable of reducing the subgingival bacterial flora to such a low level that gingival inflammation decreased and keratinization occurred, apparently as the result of lowered mitotic activity of the sulcular epithelium.

All plaque control regimes significantly reduced bacterial plaque formation compared to pretreatment plaque measurements. For those regimes associated with relatively greater plaque deposition, it may be that the routine and thorough removal of bacterial deposits was enough to allow the sulcular epithelium to fully differ- entiate and show its potential to keratinize. Regular plaque removal would inhibit maturation of the bacterial flora and maintain the healthy sulcular environment which had been established initially for all experimental regimes by means of a thorough prophylaxis followed by a period of parenteral antibiotic therapy. The frequent rubber cup prophylaxes may also have had a mechanical effect in stimulating the sulcular epithelium to keratinize. This would be in accord with the report that, in humans, ${ }^{13}$ intrasulcular toothbrushing may cause the sulcular epithelium to keratinize. However, in the present study, the mechanical factor alone may not have been responsible for the observed sulcular keratinization because even in the absence of frequent prophylaxes, other effective regimes of plaque control permitted sulcular keratinization.

All experimental regimes significantly reduced their respective Inflammatory Indices relative to the Control values. In general, with the Crestal Inflammatory Indices of the various experimental regimes arranged in increasing order of magnitude, an inverse relationship was found when compared to a similar ordering according to Keratin Widths and Keratin Lengths. Furthermore, a significant negative correlation was found between the Crestal Inflammatory Indices and the Keratin Widths. A negative correlation was also apparent between the Crestal Inflammatory Indices and Keratin Lengths, although it was not statistically significant. A significant positive correlation was found between Keratin Width and Keratin Length. These findings are in direct accordance with the reported association between reduced gingival inflammation and sulcular keratinization. ${ }^{3}$ However, it should be realized that the value of the correlation coefficient is important when that value is near 0.9, because such a high value permits the prediction of one 
variable from the known value of the other variable with an $81 \%(0.9 \times 0.9)$ reduction in variability. With correlation coefficient values less than 0.9 , the positive or negative nature of the correlation coefficient deserves particular consideration. Thus, while a significant negative correlation coefficient $(-0.3570)$ was found between Crestal Inflammatory Indices and Keratin Widths, and the correlation coefficient $(-0.1366)$ between Crestal Inflammatory Indices and Keratin Length lacked significance, both values were low and the direction of the sign assumes importance. Where the lingual Keratin Width was found to be significantly greater than the corresponding buccal Keratin Width for the same regime, the lingual Crestal Inflammatory Index was reduced compared to the buccal Crestal Inflammatory Index. However, the buccal and lingual differences in Crestal Inflammatory Index were not large enough to explain the marked differences in buccal and lingual Keratin Widths, and it may be that individual differences exist regarding the propensity of the sulcular epithelium to keratinize.

It has been well established that a nonkeratinized sulcular epithelium is permeable to a variety of materials, including tritium labelled albumen (in guinea pigs), ${ }^{14}$ tritiated $E$. coli endotoxins (in dogs) ${ }^{15}$ and tritiated bovine hyaluronidase (in marmosets). ${ }^{16}$ However, in the latter investigation, a pilot study performed in rats showed that gingival sulcular keratinization acted as a definite barrier to hyaluronidase penetration. Additionally, a study in hamsters ${ }^{17}$ demonstrated that following a topical application of tritiated thymidine, a significantly greater proportion of labelled basal cells was found for the nonkeratinized sulcular epithelium than for the keratinized attached gingiva.

Thus, normally keratinized epithelia have been found to resist the penetration of topically applied substances, while normally nonkeratinized sulcular epithelia apparently lack a surface layer resistant to penetration. In this investigation, various plaque control regimes permitted the transformation of a normally nonkeratinizing sulcular epithelium to a keratinizing sulcular epithelium. It would be interesting to know if such plaque-controlled sulcular keratinization would demonstrate also a barrier to penetration like that of a normally keratinized epithelium. Enhanced sulcular resistance to diffusible substances could be of considerable importance in regard to resisting the development and progression of periodontal disease.

\section{CONCLUSIONS}

Within the limits of this study, it may be concluded that:

1. All experimental combinations of prophylaxes, topical chlorhexidine and systemic tetracycline caused a reduction in plaque deposition and gingival inflammation.
2. All of the plaque control regimes reduced gingival inflammation sufficiently to permit the keratinizing potential of the gingival sulcular epithelium to express itself.

3. The greater the reduction in crestal inflammation, the greater the width and length of the keratinized sulcular epithelium. As the width of the keratinized sulcular surface increases, the distance that the keratinization extends into the sulcus also increases.

\section{REFERENCES}

1. Caffesse, R. G., Karring, T., and Nasjleti, C. E.: Keratinizing potential of the sulcular epithelium. $J$ Periodontol 48: 140, 1977.

2. Bral, M. M., and Stahl, S. S.: Keratinizing potential of human crevicular epithelium. J Periodontol 48: 381, 1977.

3. Caffesse, R. G., Kornman, K. S., and Nasjleti, C. E.: The effect of intensive antibacterial therapy on the sulcular environment in monkeys. Part II: Inflammation, mitotic activity and keratinization of the sulcular epithelium. J Periodontol 51: 155, 1980.

4. Löe, H., and Silness, J.: Periodontal disease in pregnancy. 1 . Prevalence and severity. Acta. Odontol. Scand., 21: 533, 1963.

5. Silness, J. and Löe, H.: Periodontal disease in pregnancy. II. Correlation between oral hygiene and periodontal condition. Acta Odontol Scand 221: 121, 1964.

6. Kornman, K. S., Caffesse, R. G., and Nasjleti, C. E.: The effect of intensive antibacterial therapy on the sulcular environment in monkeys. Part I: Changes in the bacteriology of the gingival sulcus. $J$ Periodontol 51: 34, 1980.

7. Caffesse, R. G., Nasjleti, C. E., and Castelli, W. A.: The role of sulcular environment in controlling epithelial keratinization. $J$ Periodontol 50: 1, 1979.

8. Karring, T., Östergaard, E., and Löe, H.: Conservation of tissue specificity after heterotopic transplantation of gingiva and alveolar mucosa. J Periodont Res 6: 282, 1971.

9. Karring, T., Lang, N. P., and Löe, H.: The role of gingival connective tissue in determining epithelial differentiation. $J$ Periodont Res 10: 1, 1975.

10. Jarrett, A.: The Physiology and Pathophysiology of the Skin, vol. 1. Academic Press, 1973.

11. Squier, C. A., Johnson, N. W., and Hackemann, M.: Structure and function of normal human oral mucosa. A. E. Dolby (ed), Oral Mucosa in Health and Disease. Springfield, IL, Charles Thomas, 1975.

12. Abrams, G. C., Bauer, H., and Sprinz, H.: Influence of the normal flora on mucosal morphology and cellular renewal in the ileum. A comparison of germ-free and conventional mice. Lab Invest 12: 355 , 1963.

13. Fry, H. R., and App, G. R.: Histologic evaluation of the effect of intrasulcular toothbrushing on human sulcular epithelium. $J$ Periodontol 49: 163, 1978 .

14. Tolo, K.: A study of permeability of gingival pocket epithelium to albumin in guinea pigs and Norwegian pigs. Arch Oral Biol 16: 881, 1971.

15. Schwartz, J., Stinson, F., and Parker, R.: The passage of tritiated bacterial endotoxin across intact gingival crevicular epithelium. $J$ Periodontol 43: 270, 1972.

16. Caffesse, R. G., and Nasjleti, C. E.: Enzymatic penetration through intact sulcular epithelium. J Periodontol 47: 391, 1976.

17. Jensen, R. L., and Folke, L. E.: The passage of exogenous tritiated thymidine into gingival tissues. $J$ Periodontol 45: 786, 1974.

Send reprint requests to Dr. Raul G. Caffesse, Department of Periodontics, The University of Michigan School of Dentistry, Ann Arbor, MI 48109. 